\title{
Method for Mixed Gas Detecting in Coal Mine Based on Improved RBF Neural Network
}

\author{
Ruiqing $\mathrm{Mao}^{1, \star}$, Xiliang $\mathrm{Ma}^{1,2}$ and Yong Wang ${ }^{2}$ \\ ${ }^{1}$ Jiangsu Key Laboratory of Large Engineering Equipment Detection and Control, Xuzhou Institute of Technology, 221111, China \\ ${ }^{2}$ School of Mechanical and Electrical Engineering, China University of Mining and Technology, Xuzhou,Jiangsu,221116,China
}

\begin{abstract}
Aiming at the problem of the coal mine rescue robot's perception of toxic and harmful gas in Coal Mine, The cross sensitivity of partial gas is reduced by using double gas sensor, and the influence of the variable factors such as temperature and humidity is considered, The improved RBF neural network based on genetic neural network algorithm and $\mathrm{K}$ clustering algorithm is proposed in combination with the practical application of coal rescue robot, A hybrid gas detection system based on RBF neural network is built. The experimental results show that: the improved RBF neural network algorithm is applied to the training of mixed gas quantitative recognition, The convergence speed is faster than the RBF neural network algorithm, and the learning accuracy is higher, Improve the performance of RBF and the detection accuracy of the mixed gas detection system, the system can take the quantitative detection of H2S, $\mathrm{CO}, \mathrm{CO} 2$ and $\mathrm{CH} 4$ four kinds of gases and their mixed gas in the detection range.
\end{abstract}

\section{Introduction}

Coal mine environment is complex and bad, containing a large number of toxic and harmful gases such as methane, carbon monoxide, carbon dioxide, etc., in the coal mining, transportation, processing and utilization, such as pollution and safety problems, the body and mind have a great impact on the staff. One of the main tasks of mine rescue robot is to detect the environmental information such as gas, temperature and humidity, so as to make accurate and timely assessment of the complex and dangerous environment information, and then make a control decision, to achieve autonomous mobile, personnel tracking, rescue and so on. Therefore, the coal mine rescue robot can accurately detect the gas has become a key technology of the rescue robot environment perception system. However, in the detection of toxic and harmful mixed gas, the main component of the gas can be weakened or lost due to the interference of various gases, which can lead to the detection and control of the rescue robot.

At present, many scholars have done some research on the detection method of mixed gas, Literature [1]pointed out that the Bayesian decision can make up for the deficiency of single gas detection, and the CO and H2S mixed gas concentrations are detected by using three kinds of gas sensor, which is based on the combination of single sensor and multi sensitive; Literature [2] constructed a can reduce the response to cross PCA RBF neural network, used in photoacoustic detection output array signal processing, to solve the traditional RBF neural network in the input space serious autocorrelation detection accuracy degree of decline, accurate detection of gas volume fraction of SO2, $\mathrm{CO} 2$, CF4 gas mixture in each group; Literature [3] using differential absorption spectroscopy to measure the concentration of pollutant gases, a set of experimental apparatus, which can monitor the concentration of $\mathrm{SO} 2$ and $\mathrm{NOX}$ in the flue gas emissions, is developed; Literature [4] aiming at the problem of mixed gas detection, using the error back propagation (BP) algorithm and genetic algorithm, the method of adaptive genetic algorithm to optimize the BP neural network is proposed to achieve quantitative detection; Literature [5] is developed based on AlN (aluminum nitride semiconductor four array sensor, MEMS microstructure of several kinds of gas and mixed gas is rapid and accurate on-line detection by using artificial neural network pattern recognition as a gas at the core of the method. Literature [6] introduced the multiple sensor detection of gas mixture concentration of components of linear principle and method. Literature [7] the integration of the BP neural network is adopted to the response of the harmful gas sensor array of three kinds of mixed signal by regression analysis, in order to improve the prediction accuracy of integration BP neural network, and using Adaboost algorithm for integration of the BP neural network is optimized. Literature [8] with adaptive genetic algorithm is proposed to optimize the $\mathrm{BP}$ neural network method to realize the quantitative detection of mixed gas; Literature [9] choose support vector machine (SVM) regression algorithm, dynamic detection of hydrogen (H2) and methane (CH4) set of mixed gas.

To some extent, the research on the problem of mixed gas detection is solved, many using sensor array with pattern recognition and neural network to test the mixed gas, but did not consider the actual online detection of gas mixture, also did not consider in the coal mine actual application background and the complexity, the effects of variables such as temperature and humidity, resulting in online detection of gas when the result is bad; There are scholars using some of the characteristics of light decomposition components of gas infrared absorption bands, however when using photoacoustic test to detect the component has serious cross response, influence the accuracy of detection. According to the above problem, this paper adopts double gas sensor to reduce part of the gas cross sensitivity, at the same time, combined with practical application of coal mine rescue robot is put forward based on GA optimization and k improved RBF neural network clustering algorithm, improve the performance of RBF and the prediction precision of the system. 


\section{The working principle and composition of the mixed gas detection system}

The mixed gas detection system consists of a gas sensor array, signal processing system and pattern recognition system, and its structure is shown in Figure 1. Gas sensor array is composed of a variety of different types of sensors array to form its "sensory" device. Signal processing system for the sensor array response mode to pre process, complete filtering, switching and feature extraction, and so on. Pattern recognition subsystem uses the corresponding algorithm to detect the characteristic parameters of the model.

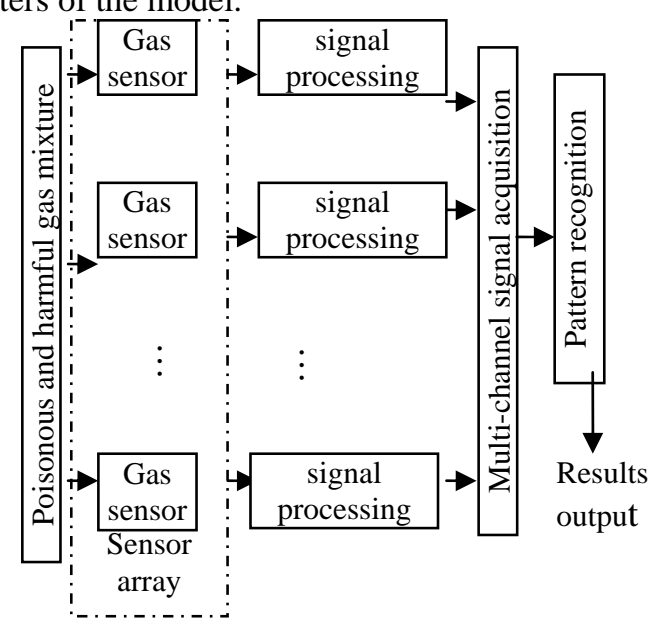

Figure 1. Schematic diagram of the mixed gas detection system

\section{Improved RBF neural network learning method}

K-means clustering method is the most classic and most common learning algorithm of RBF neural network, the main idea is as follows: the input of the sample is $X_{1}, X_{2}, \cdots X_{n}$, the corresponding target output is $Y_{1}, Y_{2}, \cdots Y_{n}$, the RBF neural network in the $\mathrm{J}$ of the activation function $\Phi_{j}(*)$ of the hidden nodes. $\mathrm{K}$ is the number of iterations, so that the clustering center of the first $\mathrm{k}$ iteration is $h_{1}(k), h_{2}(k), \cdots h_{n}(k)$, the corresponding clustering domain is $W_{1}(k), W_{2}(k), \cdots W_{n}(k)$.

Using K-means clustering algorithm to determine the RBF network data center and the expansion constant of the steps are as follows:

(1) Initialization of the algorithm: select the initial cluster centers of $\mathrm{h}$ different, and make $\mathrm{k}=1$. There are many methods for the initial clustering center, for example, the random selection from the sample input, or the selection of the $\mathrm{H}$ sample input, but the $\mathrm{h}$ initial data center must take different values.

(2)The Euclidean distance between the sample input and clustering center:

$$
\square x_{j}-h_{i}(k) \square, i=1,2, \cdots, h, j=1,2, \cdots, n
$$

(3) To classify the sample input by the minimum distance principle.

$$
i\left(X_{j}\right)=\min \square x_{j}-h_{i}(k) \square, i=1,2, \cdots, h
$$

$X_{j}$ is classified into category i, $X_{j} \subset w_{i}(k)$

(4) The clustering center of the recalculation of all hidden nodes:

In the formula, $N_{i}$ is the number of samples contained in the ${ }^{W_{j}}(k)$ cluster.

(5)If $h_{i}(k+1) \neq h_{i}(k)$, go to step (2), otherwise go to step (6), clustering center no longer changes, stop clustering.

6) According to the clustering center determines the distance between each expansion constants of the hidden nodes. The expansion constant of the hidden nodes is $\delta i=\kappa d_{i}$, which ${ }^{d_{i}}$ is the minimum distance between the i-th data centers and other data centers, $d_{i}=\min \square h_{i}-h_{i}(k) \square, \quad \kappa$ is the overlap coefficient.

Genetic algorithm is adopted to optimize the weights and bias of RBF neural network, in order to achieve the purpose of improving the recognition rate of RBF neural network and reducing the number of hidden layer neurons of RBF, the steps are as follows:

(1) K mean clustering is used to get the data center and the expansion constant of the real number of encoding to produce the population, the initialization of the chromosome.

(2) Using training samples to train the parameters, calculate the fitness value of each chromosome.

(3)test termination conditions are satisfied, if satisfied, then the loop terminates, optimal output parameters, calculate the output value, such as do not meet the execute step (4).

(4) implementation of genetic manipulation, selection, crossover, mutation operation.

(5)The corresponding prediction algorithm to predict the values of the variables in the process of utilization, better prediction of chromosome.

6) second judgment, if meet the termination conditions are end loop, or jump to step (2)

The improved RBF neural network algorithm flow is shown in Figure 2. 


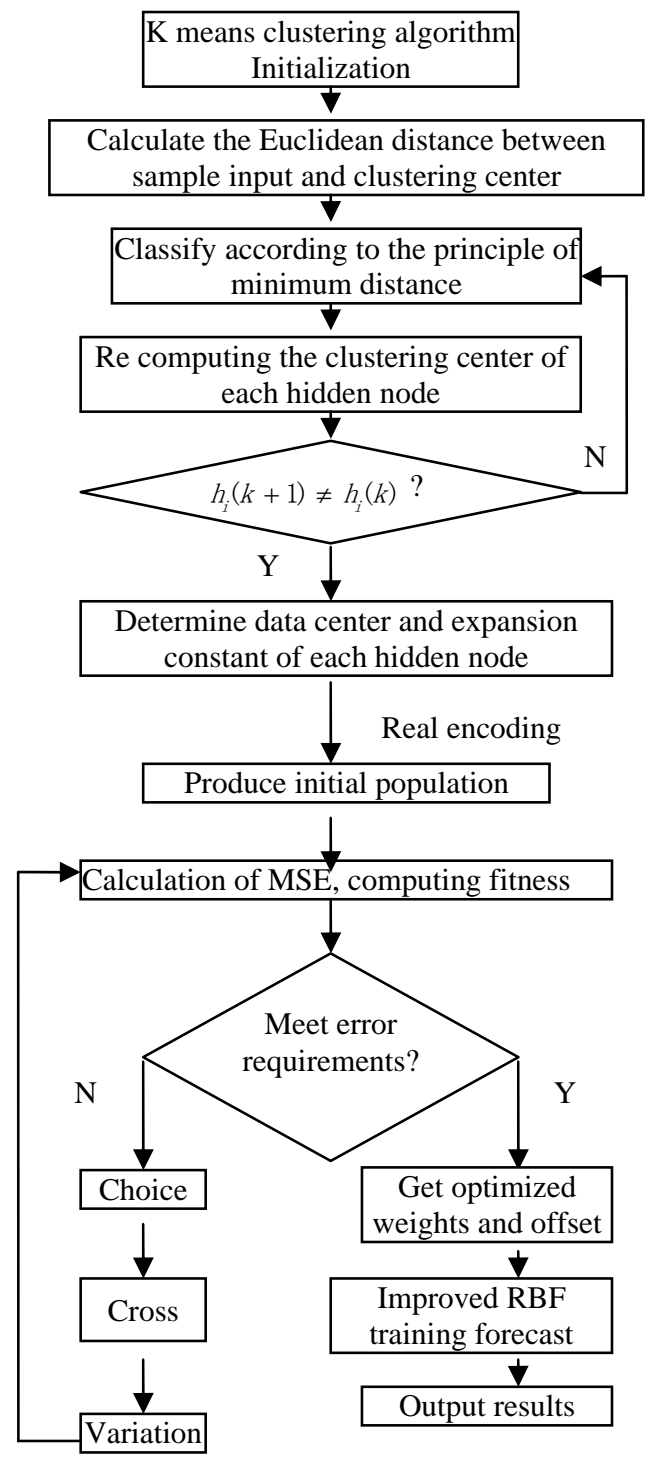

Figure 2. improved RBF neural network algorithm proces

\section{4 experiments and analysis}

\section{1 experimental procedure}

According to coal mine safety regulations and sensor detection range of selected gas volume fraction range: $\mathrm{CH} 4[0 \sim 5000 \mathrm{ppm}], \mathrm{H} 2 \mathrm{~S}[0 \sim 200 \mathrm{ppm}], \mathrm{CO}[0 \sim$ 1000ppm], CO2[0 $5000 \mathrm{ppm}], 500$ groups of mixed gas and experiment, covering the whole of the measured concentration space, provide a suitable sample for neural network. In this experiment, five kinds of gas sensors were used to make the sensor array, and the mixed gas was configured. After a period of time, the data is collected, and the data is normalized, and the neural network is trained by the improved RBF neural network. In the process of training, the network parameters of RBF neural network are trained by repeated training. In the test phase, the remaining test sample is entered into the training good network, and the accuracy of the whole system is tested by comparing the test results with the real value.

\section{2 experimental results and analysis}

In the experiment, the output signal and temperature and humidity of the 4 kinds of gas sensor array, so the neural network input layer is 6 , the output layer neuron is $4 \mathrm{RBF}$ neural network hidden layer neuron number is 13 , the optimized target error is 0.001 , the maximum iteration number is 1000 . The collected data of the 165 groups were normalized, and the data of the 150 groups were randomly selected as the training sample to train the RBF neural network and the improved RBF neural network. After training, the network parameters were saved. The remaining 15 sets of data were input into the trained network to detect the accuracy of neural network prediction. The test results were Table 1 and Table 2 respectively. RBF neural network and improved RBF neural network in the error curve of the iterative process shown in (a) and (b) of Figure 3, it is clear that the improved RBF neural network is very fast convergence and achieve the target error.

Table 1. Test results of RBF neural network

\begin{tabular}{|c|c|c|c|c|}
\hline$\underset{\text { gas }}{\text { Mixed }}$ & $\mathrm{CO} 2$ & H2S & CO & CH4 \\
\hline $\mathrm{S} / \mathrm{N}$ & \multicolumn{3}{|c|}{ RBF neural network output value(PPM) } & Expected value of Test sample(PPM) \\
\hline \multirow{2}{*}{1} & 435.723 & 0.000 & 1.988 & 329.96 \\
\hline & 400 & 0 & 0 & 300 \\
\hline \multirow[t]{2}{*}{2} & 48.984 & 0.000 & 1.753 & 149.656 \\
\hline & 50 & 0 & 0 & 150 \\
\hline \multirow{2}{*}{3} & 0.000 & 31.673 & 0.000 & 0.000 \\
\hline & 0 & 33.3 & 0 & 0 \\
\hline \multirow{2}{*}{4} & 7.890 & 54.502 & 2.098 & 0.000 \\
\hline & $\begin{array}{l}0 \\
0.000\end{array}$ & $\begin{array}{l}60 \\
3.287\end{array}$ & $\begin{array}{l}0 \\
20.000\end{array}$ & $\begin{array}{l}0 \\
0.003\end{array}$ \\
\hline 5 & 0 & 0 & 20 & 0 \\
\hline 6 & $\begin{array}{l}451.708 \\
500\end{array}$ & $\begin{array}{l}16.286 \\
10\end{array}$ & $\begin{array}{l}91.447 \\
100\end{array}$ & $\begin{array}{l}51.137 \\
50\end{array}$ \\
\hline \multirow{2}{*}{7} & 0.000 & 0.000 & 0.000 & 20.641 \\
\hline & 0 & 0 & 0 & 20 \\
\hline \multirow{2}{*}{8} & 106.641 & 21.182 & 0.000 & 221.563 \\
\hline & 100 & 20 & 0 & 200 \\
\hline \multirow{2}{*}{9} & 232.387 & 84.002 & 5.062 & 102.918 \\
\hline & 200 & 100 & 0 & 100 \\
\hline \multirow{2}{*}{10} & 60.957 & 0.000 & 37.294 & 489.232 \\
\hline & 50 & 0 & 40 & 500 \\
\hline \multirow{2}{*}{11} & 333.169 & 0.0193 & 66.330 & 3.159 \\
\hline & 333.3 & 0 & 66.6 & 0 \\
\hline \multirow{2}{*}{12} & 4.598 & 20.956 & 22.867 & 423.524 \\
\hline & 0 & 20 & 20 & 400 \\
\hline \multirow{2}{*}{13} & 5.264 & 90.120 & 33.744 & 0.002 \\
\hline & 0 & 100 & 40 & 0 \\
\hline \multirow{2}{*}{14} & 152.964 & 67.265 & 16.174 & 0.005 \\
\hline & 150 & 60 & 10 & 0 \\
\hline \multirow{2}{*}{15} & 332.226 & 31.601 & 67.885 & 000.3 \\
\hline & 300 & 26.6 & 60 & 0 \\
\hline
\end{tabular}


Table 2. Test results of improved RBF neural network

\begin{tabular}{|c|c|c|c|c|}
\hline $\begin{array}{l}\text { Mixed } \\
\text { gas }\end{array}$ & $\mathrm{CO} 2$ & H2S & CO & CH4 \\
\hline \multirow[t]{2}{*}{$\mathrm{S} / \mathrm{N}$} & $\begin{array}{l}\text { Improved } \\
\text { (PPM) }\end{array}$ & RBF neural & network & output value \\
\hline & \multicolumn{4}{|c|}{ Expected value of Test sample(PPM) } \\
\hline \multirow{2}{*}{1} & 405.145 & 0.000 & 1.002 & 309.65 \\
\hline & 400 & 0 & 0 & 300 \\
\hline \multirow{2}{*}{2} & 49.238 & 0.000 & 0.958 & 150.235 \\
\hline & 50 & 0 & 0 & 150 \\
\hline \multirow{2}{*}{3} & 0.000 & 32.501 & 0.000 & 0.000 \\
\hline & 0 & 33.3 & 0 & 0 \\
\hline \multirow[b]{2}{*}{4} & 0.520 & 57.673 & 1.09 & 0.000 \\
\hline & 0 & 60 & 0 & 0 \\
\hline \multirow{2}{*}{5} & 0.000 & 3.287 & 20.000 & 0.002 \\
\hline & 0 & 0 & 20 & 0 \\
\hline \multirow{2}{*}{6} & 486.651 & 13.182 & 97.741 & 50.37 \\
\hline & 500 & 10 & 100 & 50 \\
\hline \multirow{2}{*}{7} & 0.000 & 0.000 & 0.000 & 20.132 \\
\hline & 0 & 0 & 0 & 20 \\
\hline \multirow{2}{*}{8} & 102.465 & 21.287 & 0.000 & 210.514 \\
\hline & 100 & 20 & 0 & 200 \\
\hline \multirow{2}{*}{9} & 205.562 & 92.209 & 3.602 & 101.181 \\
\hline & 200 & 100 & 0 & 100 \\
\hline \multirow{2}{*}{10} & 52.448 & 0.000 & 39.265 & 496.345 \\
\hline & 50 & 0 & 40 & 500 \\
\hline \multirow{2}{*}{11} & 334.092 & 0.0193 & 66.203 & 2.348 \\
\hline & 333.3 & 0 & 66.6 & 0 \\
\hline \multirow{2}{*}{12} & 2.008 & 20.658 & 21.612 & 401.989 \\
\hline & 0 & 20 & 20 & 400 \\
\hline \multirow{2}{*}{13} & 1.028 & 93.601 & 37.812 & 0.001 \\
\hline & 0 & 100 & 40 & 0 \\
\hline \multirow{2}{*}{14} & 149.95 & 61.147 & 11.359 & 0.002 \\
\hline & 150 & 60 & 10 & 0 \\
\hline \multirow{2}{*}{15} & 312.651 & 29.375 & 64.581 & 0.001 \\
\hline & 300 & 26.6 & 60 & 0 \\
\hline
\end{tabular}

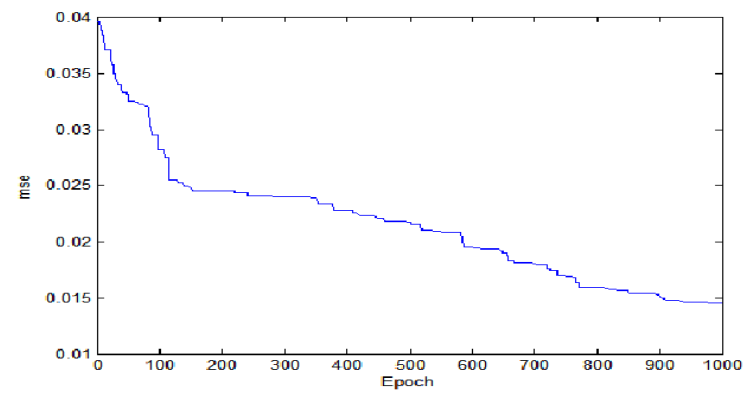

(a) Training results of RBF neural network

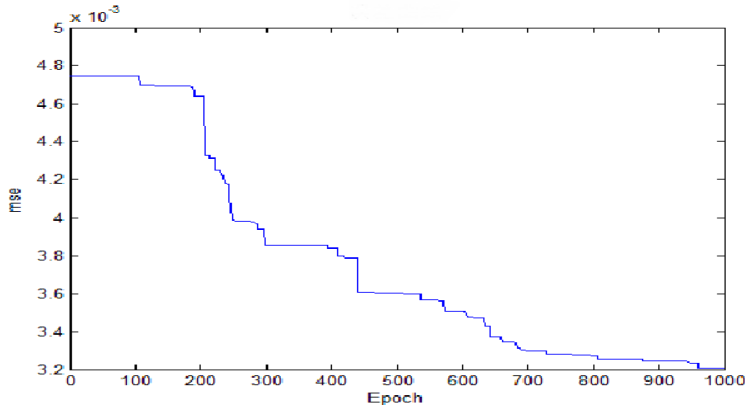

(b) Training results of improved RBF neural network

Figure 3. error curve contrast of RBF neural network and improved RBF neural network
When compared to Table 1 and Table 2, it can be seen that in the same training samples, the improved RBF neural network has much smaller prediction error than the RBF neural network, The average relative error of the improved RBF neural network is less than 5\%; In network training, the improved RBF neural network is better than RBF neural network, which can avoid the local optimal solution effectively and improve the stability of the forecasting system.

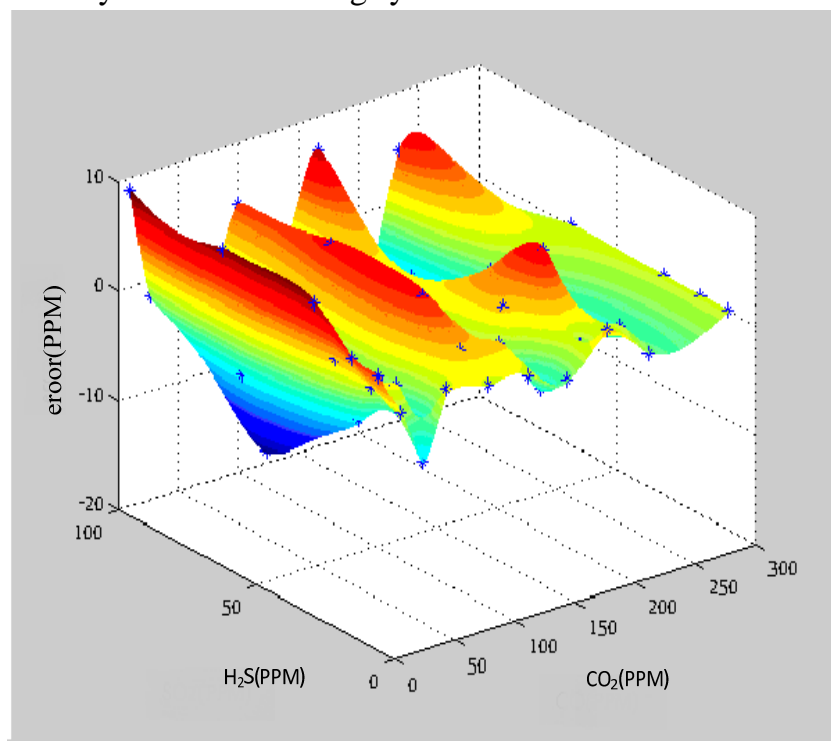

Figure 4. Improved RBF for quantitative detection of $\mathrm{CO} 2$ and $\mathrm{H} 2 \mathrm{~S}$ mixed gas

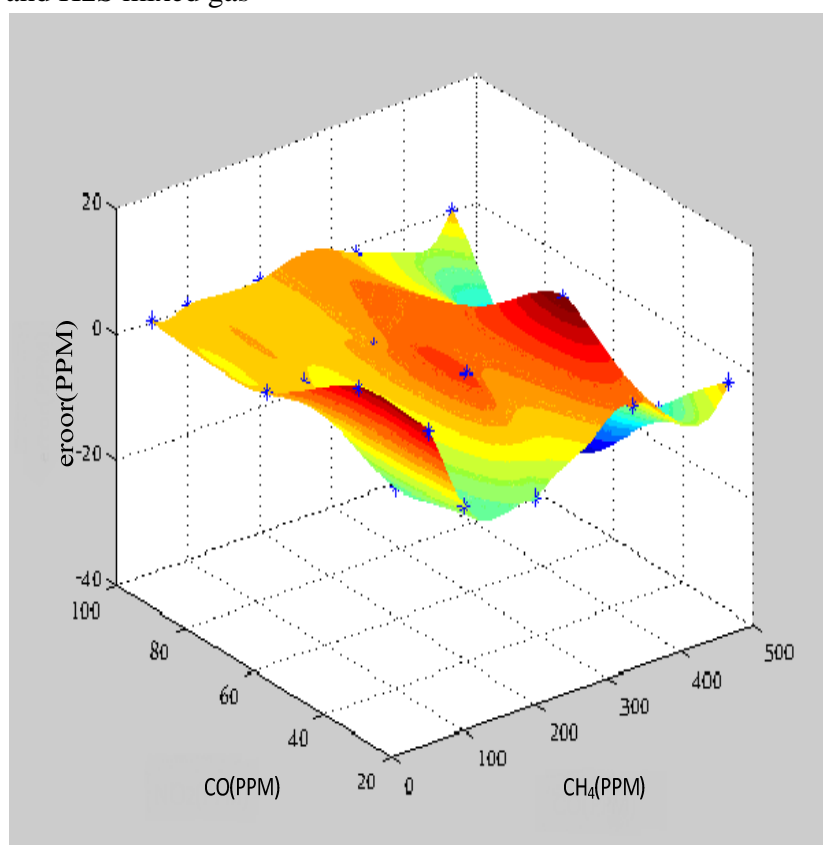

Figure 5. the Improved RBF for quantitative detection of $\mathrm{CO}$ and $\mathrm{CH} 4$ mixed gas

In order to improve the quantitative detection error of RBF neural network, this paper takes CO2 and H2S, CO and $\mathrm{CH} 4$ as an example to draw the error three dimensional surface map. The error surfaces of the CO2 and H2S mixed gas are shown in Figure 4, and the surface of the mixed gas of $\mathrm{CH} 4$ and $\mathrm{CO}$ is shown in Figure 5. In the picture, the $\mathrm{X}$ and $\mathrm{Y}$ axes are two kinds of gas concentration, and the $\mathrm{Z}$ axis is the measurement error value. Through the analysis of the error surface, it is 
known that the system is more accurate for the detection of $\mathrm{CO}$ and $\mathrm{CH} 4$ mixture gas, On the whole, the testing error of the system meets the design requirements, that is, the detection range is within the limits specified. Thus, the system can take the quantitative detection of $\mathrm{CO} 2$, $\mathrm{H} 2 \mathrm{~S}, \mathrm{CO}$ and $\mathrm{CH} 4$ four kinds of gases and their mixed gas.

\section{5 conclusions}

In view of the problem of the detection of toxic and harmful gases in coal mines, this paper uses a double gas sensor to reduce the cross sensitivity of the gas, and the effects of temperature, humidity and other factors, and combined with the practical application of coal mine rescue robot based on GA optimization and K clustering algorithm to improve the RBF neural network, improve the performance of RBF and hybrid gas detection system prediction accuracy. From the experimental results, we can get the following conclusions: using sensor array to detect the effect of mixed gas can eliminate gas cross response, the improved RBF neural network and sensor array technology combined detection system has achieved good results in the detection of toxic and harmful gases in coal mine. In the detection range, the system can take the quantitative detection of $\mathrm{CO} 2, \mathrm{CO}$, $\mathrm{H} 2 \mathrm{~S}$ and $\mathrm{CH} 4$ four kinds of gases and their mixed gas.

\section{References}

[1] Mao RuiQing. Multi-channel simultaneous data acquisition system with variable sampling rate Applied Mechanics and Materials, 635-637:896-900 (2014)

[2] Sun Y, Zhang L B, Gu X S. A hybrid co-evolutionary cultural algorithm based on particle swarm optimization for solving global optimization problems, Neuro computing, 98: 76-89(2012).

[3] Wang H F, Moon I, Yang S X, et al. A memetic particle swarmoptimization algorithm for multimodal optimization problems, Information Sciences, 197: 38 -52(2012)

[4] Mao RuiQing, Ma XiLiang. Design of the intrinsic safety CO monitor with low power consumption, Applied Mechanics and Materials, 635-637:886-889. (2014)

[5] Tatsuhiro Yoshigoe, Tatsuo Nishina, Susumu SATO, etc. Development of gas seneor rorvolatail organic compunds (VOCs) by using of solid polymer electrolyte membrane electrodes,Analytical Sciences, (17):1023-1025. (2001)

[6] De Vito S, E. Massera, M. Piga, etc. On field calibration of an electronic nose for benzene estimation in an urban pollution monitoring scenario, Sensors and Actuators B-Chem, (129): 750-757(2008).

[7] KABIR H, WANG Y, YU M, etc. Neural network inverse modeling and applications to microwave filter design, IEEE Transactions on Microwave Theory and Techniques, 56(4): 867879(2008).
[8] Bipan Tudu, Arun Jana, Animesh Metla, etc. Electonic nose for black tea quality evaluation by an incremental RBF network, Sensors and Actuators B: Chemical, 138(1): 90-95(2009).

[9] Barbu T L, Vinogradov I, Durry G, et al. TDLAS a laser diode sensor for the in situ monitoring of $\mathrm{H} 2 \mathrm{O}$, $\mathrm{CO} 2$ and their isotopes in the Martian atmosphere, Advances in Space Research, 38(4): 718-725(2006). 